\title{
HARP D111-136 enhances radiation-induced apoptosis of U87MG glioblastoma by induction of the proapoptotic protein CHOP
}

\author{
RACHA KARAKY ${ }^{1}$, EMILIE GOBBO ${ }^{1}$, PAULE OPOLON ${ }^{1}$, JEAN DELBÉ ${ }^{2}$, JOSÉ COURTY $^{2}$, \\ FRANCK GRISCELLI $^{3}$, MICHEL PERRICAUDET ${ }^{1}$ and DOMINIQUE MARTEL-RENOIR ${ }^{1}$

\begin{abstract}
${ }^{1}$ CNRS, UMR 8121, Vectorologie et Transfert de Gènes, Institut Gustave Roussy, 39 rue Camille Desmoulins, Villejuif; ${ }^{2}$ CNRS, EAC 7149, Laboratoire CRRET, Université Paris est, Avenue du Général de Gaulle, Créteil; ${ }^{3}$ INSERM U 745, Laboratoire de Génétique Moléculaire, Université Paris Descartes, Paris, France
\end{abstract}

Received June 8, 2010; Accepted July 13, 2010

DOI: 10.3892/ijo_00000837

\begin{abstract}
We previously demonstrated, using the glioblastoma cell line U87MG as an experimental model, that the adenoviral mediated overexpression of the truncated protein HARP $\Delta 111-136$ inhibits the proliferation of these cells in vitro as well as tumor growth and angiogenesis in vivo. This study focused on identifying the underlying mechanisms for the observed antitumoral effect. The present study demonstrated that HARP $\Delta 111-136$ induced the ATF4/ATF3/CHOP cascade resulting in a strong expression of the proapoptotic protein CHOP, leading to tumor cell apoptosis as demonstrated by PARP cleavage and FACS analysis. siRNA-mediated CHOP gene silencing abolished Ad-HARP $\Delta 111-136$ induced apoptosis. Moreover, Ad-HARP $\Delta 111-136$ increased the expression of the death receptor DR5 and enhanced U87MG cells sensitivity in vitro to TRAIL a DR5 ligand with subsequent activation of caspase 8. Infection of U87MG cells with AdHARP $\Delta 111-136$ also enhanced radiation-induced apoptosis. In vivo, the combination of Ad-HARP $\Delta 111-136$ and radiation therapy resulted in a striking inhibition $(92 \%)$ of the growth of U87MG xenografts, resulting from the potent effect on tumor angiogenesis and tumor cell apoptosis as determined by TUNEL analysis. Taken together, our results indicated that the inhibitor HARP $\Delta 111-136$ sensitized U87MG cells to apoptosis.
\end{abstract}

\section{Introduction}

Heparin affin regulatory peptide (HARP), also called pleiotrophin, is a secreted growth factor known for its oncogenic $(1)$, mitogenic (2) and angiogenic activities $(3,4)$. HARP has

Correspondence to: Dr Dominique Martel-Renoir, Laboratoire CRRET, EAC 7149 CNRS, Université Paris est, Avenue du Général de Gaulle, Créteil, France

E-mail: dominique.renoir@univ-paris12.fr

Key words: HARP/PTN, HARP inhibitors, apoptosis, CHOP, radiotherapy, angiogenesis been shown to signal through two high affinity receptors, the receptor-type protein tyrosine phosphatase $\beta / \zeta(\mathrm{RPTP} / / \zeta)$ $(5,6)$ and the anaplastic lymphoma kinase (ALK) $(7)$. HARP together with its receptors are strongly overexpressed in several tumor types particularly glioblastoma $(3,8-10)$. Glioblastoma multiforme (GBM) is the most aggressive and frequent adult primary brain tumor (11-13). Resistance to apoptosis is a well known feature of glioblastoma cells leading to a poor response to chemotherapy and radiation therapy (14). Since HARP has been considered as a growth and a survival factor for cancer cells $(15,16)$, blocking the HARP pathway could be of particular interest.

HARP $\triangle 111-136$ is a truncated form of HARP lacking the last 26 amino-acids that acts as a dominant negative effector by heterodimerization with the wild-type HARP protein $(17,18)$. In a recent study (19) we demonstrated using the human glioblastoma cell line U87MG as an experimental model, that overexpression of this truncated protein inhibited in vitro and in vivo proliferation of this tumor cell line as well as in vivo angiogenesis. A replication-defective adenovirus encoding this truncated protein (Ad-HARP $4111-136)$ was constructed and our results showed that direct intra-tumoral administration of Ad-HARP $\Delta 111-136$ inhibited growth and angiogenesis of established U87MG xenografts in nude mice. However, the underlying mechanisms by which HARP $\Delta 111$ 136 inhibits tumor proliferation remain to be explored. Herein we identified, in U87MG cells infected with Ad-HARP $\Delta 111$ 136, the activation of a set of genes ATF4/ATF3/CHOP which are components of the unfolded protein response (UPR) pathway $(20,21)$.

The UPR is primarily a survival response; however, in the case of intensive or persistent ER-stress, it triggers apoptosis resulting in cell death (22). The bZIP transcription factor $\mathrm{C} /$ EBP homologous protein (CHOP) is a critical effector of the ER stress proapoptotic arm. CHOP leads to cell cycle arrest, increases expression of a number of proapoptotic genes $(20,21)$ and decreases expression of the anti apoptotic protein $\mathrm{Bcl}-2$ $(22,23)$. In the present study, we provide the first evidence of a proapoptotic effect of the inhibitor HARP $\Delta 111-136$ on the U87MG glioblastoma cell line. Ad-HARP $\Delta 111-136$ induced a strong expression of the protein CHOP leading to increased apoptosis of this highly malignant cell line (14). Furthermore, 
a therapy combining a systemic Ad-HARP $\Delta 111-136$ administration with radiotherapy triggered massive apoptosis in established tumor xenografts in vivo.

\section{Materials and methods}

Cell culture. The human glioblastoma U87MG cell line (American Type Culture Collection, Illkirch, France) was grown in Dulbecco's modified Eagle's medium (DMEM) supplemented with $10 \%$ fetal bovine serum (FBS) (Life Technologies, Cergy Pontoise, France). When specified, cells were irradiated at a dose of 10 Gy using a $250 \mathrm{kV}$ X-Ray XSD 225 Comet tube, operating at $200 \mathrm{kV}$ and $15 \mathrm{~mA}$ with a $0.2 \mathrm{~mm} \mathrm{Cu}$-filter. This dose was chosen based on a preliminary multiple dose-response experiment.

Virus description and infection. Ad-HARPA111-136 an E1-E3deleted recombinant adenovirus that expresses the first 111 amino acids of the protein HARP was previously described (19). Adenovirus expressing no transgene (Ad-CO1) was used as control. Ad-CMV-GFP is an adenovirus expressing the green fluorescent protein (GFP), Ad-K1-5 is an adenovirus expressing the five kringles of the angiostatin (24). Adenovirus infection was carried out at a multiplicity of infection (MOI) of $500 \mathrm{PFU} / \mathrm{cell}$, in culture medium supplemented with $2 \%$ FBS

Reverse transcription polymerase chain reaction. Reverse transcription was performed from $1 \mu \mathrm{g}$ total RNA, using Thermoscrip RT-PCR system (Invitrogen) with oligo dT primer. The primers used for polymerase chain reaction are ATF3: sense 5'TGATGCTTCAACACCCAGGCC3', reverse 5'AGGGGACGATGGCAGAAGCA3'; ATF4: sense 5'AGT CGGGTTTGGGGGCTGAAG3' reverse 5'TGGGGAAA GGGGAAGAGGTTGTAA3'; ß-actin: sense 5'ACACTGT GCCCATCTACGAGG3' reverse 5'AGGGGCCGGACT CGTCATACT3'; CHOP: sense 5'GCGTCTAGAATGGC AGCTGAGTCATTGCC3' reverse 5'GCGTCTAGATCAT GCTTGGTGCAGATTC3';DR5: sense 5'CAGAGGGATTG TGTCCACCT3', reverse 5'TACGGCTGCAACTGTGA CTC3'.

Detection of apoptosis, analysis by sub-G1 flow cytometry. Both adherent and detached cells were collected and centrifuged at 1,200 rpm for $5 \mathrm{~min}$. Cells were washed with PBS, then fixed with $70 \%$ ethanol and stored at $-20^{\circ} \mathrm{C}$ overnight. Cells were washed extemporaneously with PBS and stained with propidium iodide (20 $\mu \mathrm{g} / \mathrm{ml})$ (P3566, Invitrogen, Cergy Pontoise, France) and RNase (100 $\mu \mathrm{g} / \mathrm{ml})$ (R6513, Sigma, Saint Quentin Fallavier, France) for $30 \mathrm{~min}$ at $37^{\circ} \mathrm{C}$. DNA content of individual cells was measured using a FACS Calibur apparatus and analyzed with the CellQuest program (BD Biosciences, Erembodegem, Belgium).

Western blot analysis. Pelleted cultured cells were lysed

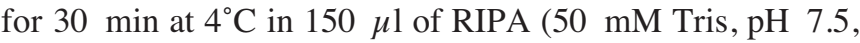
$150 \mathrm{mM} \mathrm{NaCl}, 1 \%$ deoxycholate, $1 \%$ Triton $\mathrm{X}-100,0.1 \%$ SDS, $1 \mathrm{mM}$ dithiothreitol) supplemented with complete protease inhibitor cocktail (Roche Diagnostics, Meylan, France) and anti-phosphastase cocktail (P5726, Sigma) and centrifuged $20 \mathrm{~min}$ at $11,000 \mathrm{rpm}$ to remove insoluble material.

Western blotting was conducted as previously described (18). The following primary antibodies were used: CHOP (sc-7351, diluted 1:1000; Santa Cruz Biotechnology, Montrouge, France); ATF3 (sc-188, diluted 1:1000; Santa Cruz), ATF4 (sc-200, diluted 1:1000; Santa Cruz), PTN (AF-252-PB, 1:1000; R\&D, Lille, France). Antibodies directed against: cleaved PARP $(9541,1: 1000)$, caspase 8 (9496, 1:1000), DR5 (3696, 1:3000), JNK (4668, 1:2000), p38MAPK (9211, 1:2000) were purchased from Cell Signaling (Saint Quentin en Yvelines, France). The secondary antibodies (W4021, diluted 1:30000; V8051, 1:20000 and W4011, 1:20000) were purchased from Promega (Charbonnières-lesBains, France). The conjugated HRP-ß-actin antibody (A3854, 1:50000, Sigma) was used for loading control.

RNA interference. Double-stranded RNA duplexes were purchased from Qiagen. For the knockdown of CHOP, a mix of two siRNA at a final concentration of $10 \mathrm{nM}$ was used: siRNA1, 5'GGCUCAAGCAGGAAAUCGAdTdT3' and siRNA2, 5'GGAAGUGUAUCUUCAUACAdTdT3'. The nonrelevant siRNA sequence was: 5'UUCUCCGAACGUGUCA CGUdTdT3'. Transfections were done in $10 \mathrm{~cm}$ dishes using Hiperfect transfection reagent (Qiagen) following the manufacturer's instructions.

Heparin acrylic beads. Heparin-acrylic beads (H5263, Sigma), $500 \mu 1$, were washed 5 times in PBS and incubated overnight at $4^{\circ} \mathrm{C}$ with $1 \mathrm{ml}$ of mice plasma. After 5 washes with PBS, the beads were boiled in $100 \mu \mathrm{l}$ of Western blot analysis sample buffer and resolved on SDS-PAGE.

Animals. Experiments were conducted in accordance with the recommendations of the National Institutes of Health for animal experimentation. Female NMRI nude mice (Janvier, Le Genest sur Isle, France) were used. When specified, mice received an intravenous injection of $5 \times 10^{9} \mathrm{PFU}$ of Ad-CO1 or Ad-HARP $\Delta 111-136$ in $100 \mu 1$ PBS. When specified, mice received three $\mathrm{X}$-ray irradiations, at 3 days intervals, delivered at a dose of $2.5 \mathrm{~Gy}$, using a $250 \mathrm{kV}$ X-Ray XSD 225 Comet tube operating at $200 \mathrm{kV}$ and $15 \mathrm{~mA}$ with a $0.2 \mathrm{~mm} \mathrm{Cu}$-filter.

Tumor cell inoculation in vivo. U87MG, $2 \times 10^{6}$ cells in $100 \mu 1$, were injected subcutaneously into the back of nude mice. When mean tumor volumes reached $66 \pm 20 \mathrm{~mm}^{3}$ mice were randomly assigned to each treatment group. The tumor size was monitored every two or three days by measuring the shortest (a) and the longest (b) tumor diameters with a dial caliper (b perpendicular to a) and the tumor volume was calculated as $[(a+b) / 2)] 3 \times \pi / 6(20)$. Blood samples were collected on sodium citrate and plasma were incubated $30 \mathrm{~min}$ at $56^{\circ} \mathrm{C}$ to inactivate residual viruses.

Immunostaining. Immunohistochemistry was performed as previously described $(25,26)$.

Statistics. Data were analyzed using the unpaired Student's t-test. All experiments were repeated independently at least twice. A $\mathrm{P}<0.05$ was considered to be statistically significant. 
A

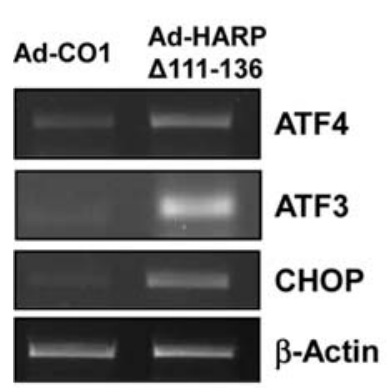

B

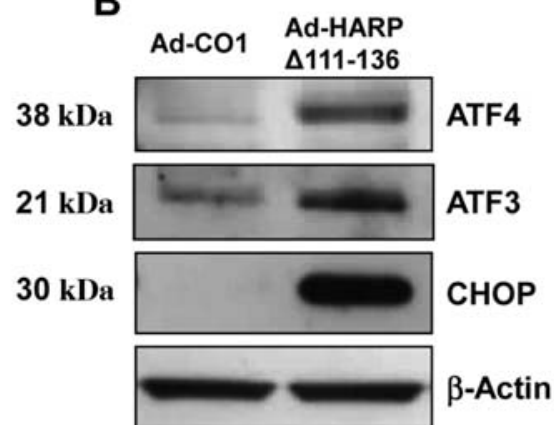

C

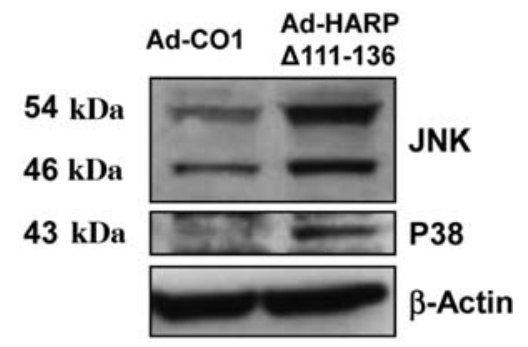

Figure 1. Ad-HARP $\Delta 111-136$ activates ER stress signaling in glioblastoma U87MG cells. (A) RT-PCR (25 cycles) was performed on $1 \mu \mathrm{g}$ of mRNA $48 \mathrm{~h}$ after Ad-HARPA111-136 or Ad-CO1 infection (500 PFU/cell). Amplification products were resolved on 1\% agarose gel and visualized by UV fluorescence. (B and C) Cell lysates were prepared $72 \mathrm{~h}$ after viral infections. Proteins, $25 \mu \mathrm{g}$, were resolved and analyzed by Western blotting using antibodies directed against ATF4 (38 kDa), ATF3 (21 kDa), CHOP (30 kDa), the phosphorylated forms of JNK, (54 and $46 \mathrm{kDa})$, p38MAPK (43 kDa). B-actin served as a loading control.

\section{Results}

Ad-HARP $\Delta 111-136$ activates ER stress signaling in glioblastoma U87MG cells. The deleted HARP peptide HARP $\Delta 111-136$ was expressed in cells using an adenovirus as described previously (19). RT-PCR was performed on RNA transcripts extracted from U87MG cells infected for $48 \mathrm{~h}$ with Ad-HARP $\Delta 111-136$ or the control Ad-CO1 virus. Results (Fig. 1A) show an increase in mRNA levels of DDIT3 (CHOP), CREB-2 (ATF4) and ATF3 genes. A rise in the levels of the proteins (Fig. 1B) ATF4, ATF3 and CHOP was demonstrated by Western blot analysis after Ad-HARP $\Delta 111$ 136 treatment.

The increase in transcript and protein levels of $C R E B-2$ (ATF4) and DDIT3 (CHOP) in Ad-HARP $1111-136$-infected U87MG cells suggests the activation of the protein kinaselike ER kinase (PERK) pathway of the ER stress signaling. Therefore, we investigated the effect of Ad-HARP $\Delta 111-136$ on the other pathways involved in ER stress signaling $(20,21)$. No change in expression was seen for ATF6 gene involved in the first pathway (data not shown). The proteins JNK, P38 MAPK were phosphorylated (Fig. 1C) after Ad-HARP $\Delta 111$ 136 infection, suggesting the activation also of the third inositol-requiring kinase 1 (IRE1) pathway.

Ad-HARP 111-136 increases expression of CHOP and apoptosis in U87MG cells. The effects of Ad-HARPA111-136 on the expression of the protein CHOP in U87MG cells were assessed by Western blotting. Our results showed that the $30 \mathrm{kDa}$ CHOP protein was strongly expressed $24 \mathrm{~h}$ after the Ad-HARP $\Delta 111-136$ infection (not shown) and maintained for up to 72 h (Fig. 2A), whereas no signal could be detected in cell lysates derived from control Ad-CO1 infected cells. CHOP has been described as a proapoptotic protein $(27,28)$ thus we examined if CHOP expression was accompanied by an increase in apoptosis of Ad-HARP $\Delta 111-136$-infected U87MG cells. Apoptosis was assessed by Western blotting using an antibody directed against the cleaved form of poly (ADP-ribose) polymerase 1 (PARP-1), the main substrate of caspase 3. As shown in Fig. 2A, cleaved PARP was found in cells infected with Ad-HARPA111-136 and not in control
Ad-CO1-infected cells indicating that Ad-HARPA111-136 expression increased apoptosis.

It is known that HARP activates protein kinase $\mathrm{C}$ (PKC) through receptor RPTPB/Z signaling pathway (29) and it has been shown that inhibition of PKC leads to increased expression of CHOP via $\beta$-catenin accumulation (30). Therefore we monitored PKC phosphorylation after Ad-HARP $\Delta$ 111-136 infection and demonstrated (Fig. 2C) that as expected overexpression of the HARP peptide HARP $\Delta 111-136$ inhibited PKC phosphorylation in U87MG cells.

In order to confirm that ER stress induction is mediated by the expression of the peptide HARP $\Delta 111-136$ and not by the forced expression of any protein in the cell, two additional controls were performed, using viruses expressing either the green fluorescent protein (Ad-GFP) or the five kringles of angiostatin Ad-(K1-5) (24). The virus Ad-(K1-5) encodes a protein which, like the peptide HARP $\Delta 111-136$ used in the present study, is a truncated and secreted protein. The absence of the expression of CHOP (Fig. 2B) in U87MG cell lysates infected with Ad-GFP or Ad-(K1-5) indicates that the observed upsurge in CHOP expression is related to the peptide HARP $\Delta 111-136$ and not to the over production of any other transgene after the viral infection.

Ad-HARPAl11-136 sensitizes U87MG cells to TRAIL-induced apoptosis. CHOP is a well-documented regulator of death receptor 5 (DR5) gene expression (31). Thus we investigated the putative role of DR5 receptor in Ad-HARP $\Delta 111-136$ induced apoptosis. Cell lysates and mRNA were prepared from Ad-HARP $\triangle 111-136$ or Ad-CO1 U87MG infected cells. The RT-PCR analysis (Fig. 3A) revealed that DR5 mRNA expression was increased in cells infected by Ad-HARP $\Delta 111$ 136. Western blotting demonstrated an increased DR5 protein expression (Fig. 3B) in U87MG cells infected with AdHARP $\Delta 111-136$. This effect was accompanied by a considerable increase of caspase- 8 cleavage, a downstream effector of DR5-induced apoptosis (Fig. 3B).

Tumor necrosis factor-related apoptosis-inducing ligand (TRAIL) is a DR5 death receptor ligand. As we demonstrated that Ad-HARP $\Delta 111-136$ infection increased DR5 expression while control virus did not, we speculated that Ad-HARP $\Delta 111$ - 


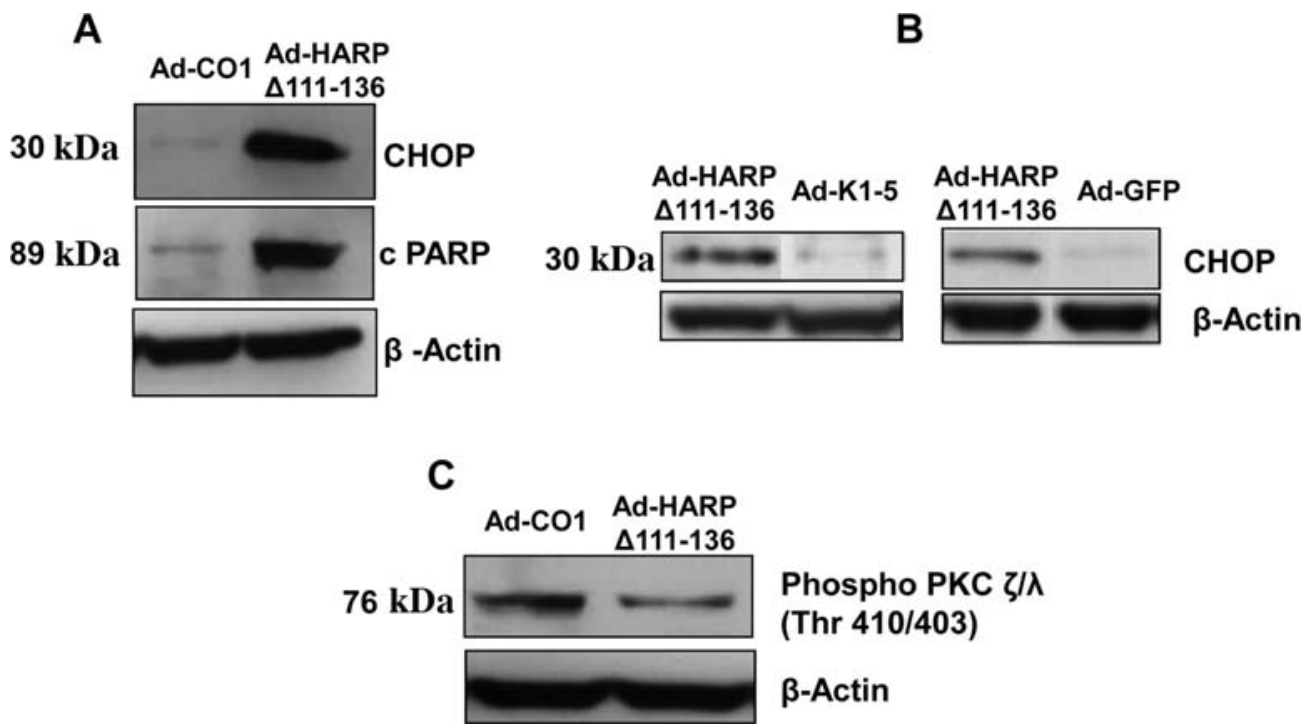

Figure 2. Ad-HARP $\triangle 111-136$ increases expression of CHOP and inhibits PKC phosphorylation in U87MG cells. U87MG cells were infected with viruses, $500 \mathrm{PFU} / \mathrm{cell}$, (Ad-CO1, Ad-HARP $\Delta 111-136$, Ad-GFP and Ad-K1-5). Cell lysates were prepared and $25 \mu \mathrm{g}$ of protein were resolved and analyzed by Western blotting using specific antibodies directed against (A and B) CHOP and (A) cleaved PARP $48 \mathrm{~h}$ after infection and (C) phosphorylated form of PKC, $18 \mathrm{~h}$ after infection. B-actin served as a loading control.

A

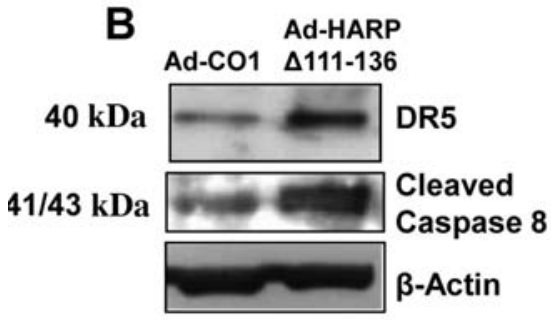

C

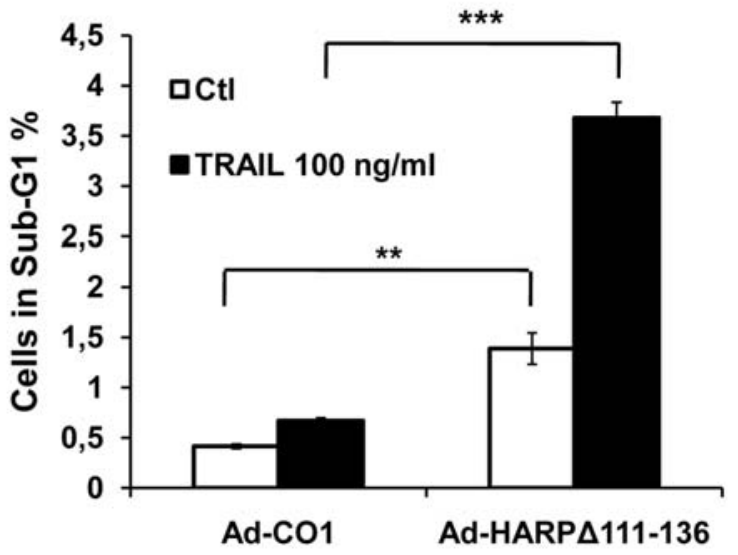

Figure 3. Ad-HARP 111 1-136 induces expression of DR5 mRNA and protein and sensitizes U87MG cells to TRAIL-induced apoptosis. (A) U87MG cells were infected with Ad-CO1 or Ad-HARP $\Delta 111-136$ viruses (500 PFU/cell), mRNA were extracted $48 \mathrm{~h}$ after infection. RT-PCR (25 cycles) for DR5 gene was performed; amplification products were resolved on $1 \%$ agarose gels. (B) U87MG cells were infected as described above. Western blot analysis was performed using the following antibodies anti-DR5 (40 kDa: mature form), $48 \mathrm{~h}$ after infection and anti-cleaved Caspase-8 (41/43 kDa), $72 \mathrm{~h}$ after infection. (C) U87MG cells were infected with Ad-CO1 or Ad-HARPA111-136. After $48 \mathrm{~h}$ TRAIL (100 $\mathrm{ng} / \mathrm{ml})$ was added to cell media; cells were incubated for another $30 \mathrm{~h}$. Cells were then stained with PI and analyzed by FACS. The sub-G1 cell population represents the apoptotic cell fraction. Values represent mean of four experiments $\pm \mathrm{SD} .{ }^{* *} \mathrm{p}<0.01,{ }^{* * *} \mathrm{p}<0.001$ Student's t-test.

136 would enhance TRAIL-induced U87MG cell apoptosis. To verify this hypothesis, U87MG cells infected with AdHARP $\triangle 111-136$ or control Ad-CO1 for $48 \mathrm{~h}$ were treated for $30 \mathrm{~h}$ with recombinant TRAIL at a final concentration of $100 \mathrm{ng} / \mathrm{ml}$. As shown in Fig. 3C, TRAIL induced apoptosis in only $0.67 \%$ of Ad-CO1-infected cells; this demonstrates the magnitude of resistance of U87MG cells to apoptosis. In Ad-HARP $\Delta 111-136$ infected cells, TRAIL induced-apoptosis was significantly enhanced $(\mathrm{p}<0.001)$ by 5 -fold compared to Ad-CO1 infected cells (Fig. 3C). Thus, Ad-HARP 111 1-136 infection sensitizes U87MG cell line to TRAIL-induced apoptosis by increasing the expression of the DR5 receptor.

Ad-HARP D111-136 enhances the radio-sensitivity of U87MG tumor cells. U87MG are known to be radiation-resistant cells, thus experiments were designed to evaluate whether the 


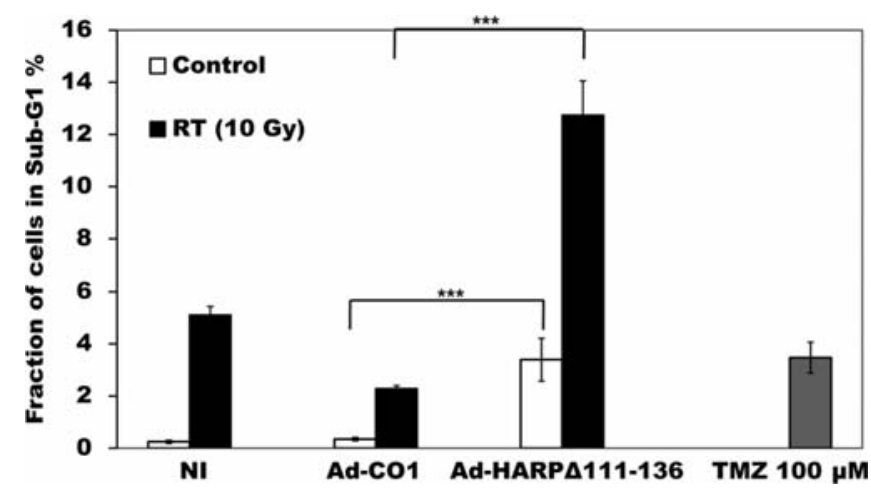

Figure 4. Ad-HARP $\Delta 111-136$ increases apoptosis of U87MG cells and enhances the apoptotic effect of radiation. U87MG cells were irradiated or not (10 Gy) then infected with Ad-HARPA111-136 or Ad-CO1 (500 PFU/cell). The cells were collected $96 \mathrm{~h}$ after infection, stained with PI and analyzed by FACS. Histograms show the percentage of sub-G1 cells estimated by FACS analysis. A positive control for apoptosis was prepared by treating U87MG with the therapeutic agent temolozomide (TMZ) and demonstrated the extent of resistance of this cell line to apoptosis. Values represent the mean of four experiments \pm SD. ${ }^{* * *} \mathrm{p}<0.001$ Student's t-test.

expression of the proapoptotic protein CHOP via AdHARP $\Delta 111-136$ could modify radiation-induced apoptosis in U87MG cells. Cultured U87MG cells were irradiated at a dose of $10 \mathrm{~Gy}$ and infected with control Ad-CO1 or AdHARP $\Delta$ 111-136 viruses. After 96 h, cells were stained with propidium iodide and apoptosis was quantified by measuring the sub-G1 population by FACS. In Fig. 4, a positive control illustrates the level of apoptosis, $3 \%$, in U87MG cells treated with $100 \mu \mathrm{M}$ of temozolomide, a cytotoxic drug. This result demonstrates the extent of resistance of this cell line to apoptosis.

No increase of the sub-G1 population could be detected after infection with Ad-CO1 (Fig. 4). Ad-HARP $\Delta 111-136$ infection significantly induced U87MG cell apoptosis: $3.5 \%$ compared with $0.3 \%$ in the Ad-CO1 control group $(\mathrm{p}<0.001)$.
Note that in all experiments, apoptosis in Ad-CO1-infected cells was less than that in non-infected cells, likely suggesting an anti-apoptotic effect of the virus by itself, and an underestimation of the HARP $\Delta 111-136$ effect. A greater apoptotic cell fraction was observed with the combination radiation and Ad-HARP $\Delta 111$-136 when compared to each therapy alone; $13 \%$ for the bitherapy compared to $2.3 \%$ for radiotherapy ( $\mathrm{p}<0.001)$, or compared to Ad-HARP $\Delta 111-136$ therapy: $3.5 \%$ $(\mathrm{p}<0.001)$. Apoptosis was clearly higher in the group that underwent dual treatment (13\%) than the sum of each treatment alone indicating a synergistic effect.

CHOP is involved in the apoptotic response of U87MG to Ad-HARPAl11-136 and radiation. In order to determine whether CHOP contributes to Ad-HARP $\Delta 111$-136-induced cell death, siRNAs were used to abolish CHOP expression after Ad-HARP $\Delta 111-136$ infection. As expected, CHOP siRNA mediated efficient knockdown of CHOP protein expression in cells infected with Ad-HARP $\Delta 111-136$ (Fig. 5A). No effect was observed with non-relevant siRNA. The effect of CHOP siRNA on the apoptosis (sub-G1 population) induced by Ad-HARP $\Delta 111-136$ infection was estimated by FACS analysis. As shown in Fig. 5B no changes in the sub-G1 population were detected in Ad-CO1 infected cells after CHOP knockdown. The use of CHOP siRNA in Ad-HARP $1111-136-$ infected cells significantly decreased $(47 \%, \mathrm{p}<0.001)$ the number of cells in the sub-G1 phase when compared with the use of non-relevant siRNA. A similar drastic and significant decline $(\mathrm{p}<0.001)$ of apoptosis was observed after knockdown of CHOP when irradiation was combined with Ad-HARP $\Delta 111$ 136 treatment. These results collectively indicate that the knockdown of CHOP in U87MG cells decreased the AdHARP $\Delta 111-136$ induced apoptosis. Taken together these results indicate that $\mathrm{CHOP}$ directly contributes to AdHARP $\Delta 111-136$ induced apoptosis.

Ad-HARPA111-136 therapy enhances the growth inhibition effect of radiation therapy on established U87MG xenografts

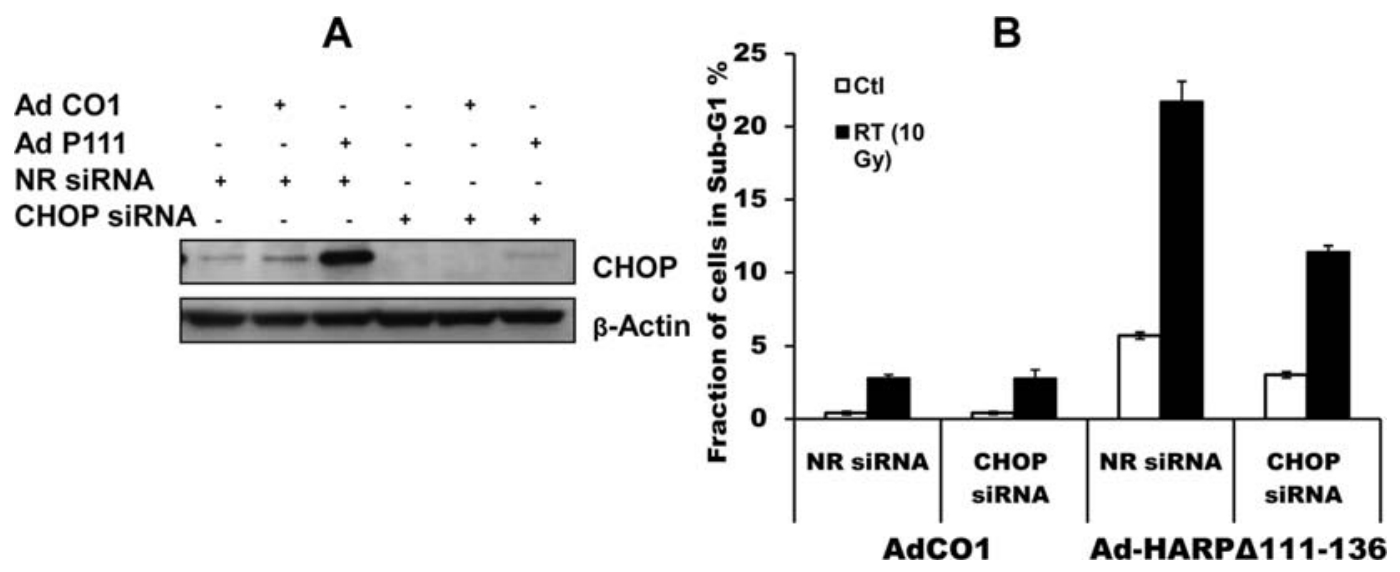

Figure 5. Ad-HARPA111-136 induced-apoptosis is dependent on CHOP. (A) Cells were transfected with 10 nM CHOP siRNA or non relevant siRNA (NR siRNA), and were infected the day after with Ad-CO1 (control) or Ad-HARP $\Delta 111-136$. Cell lysates were prepared $48 \mathrm{~h}$ after viral infection. Proteins (25 $\mu \mathrm{g}$ ) were resolved and analyzed by Western blotting using an antibody directed against CHOP. (B) U87MG cells were transfected with CHOP siRNA or nonrelevant (NR) siRNA and subjected or not to irradiation (10 Gy) and adenoviral infections. Then, 96 h after viral infection, cells were fixed, stained with PI and subjected to FACS analysis. Histograms represent the percentage of apoptotic cells in each experimental condition. Values are mean of four experiments \pm SD. p $<0.001$ Student's t-test. 
A

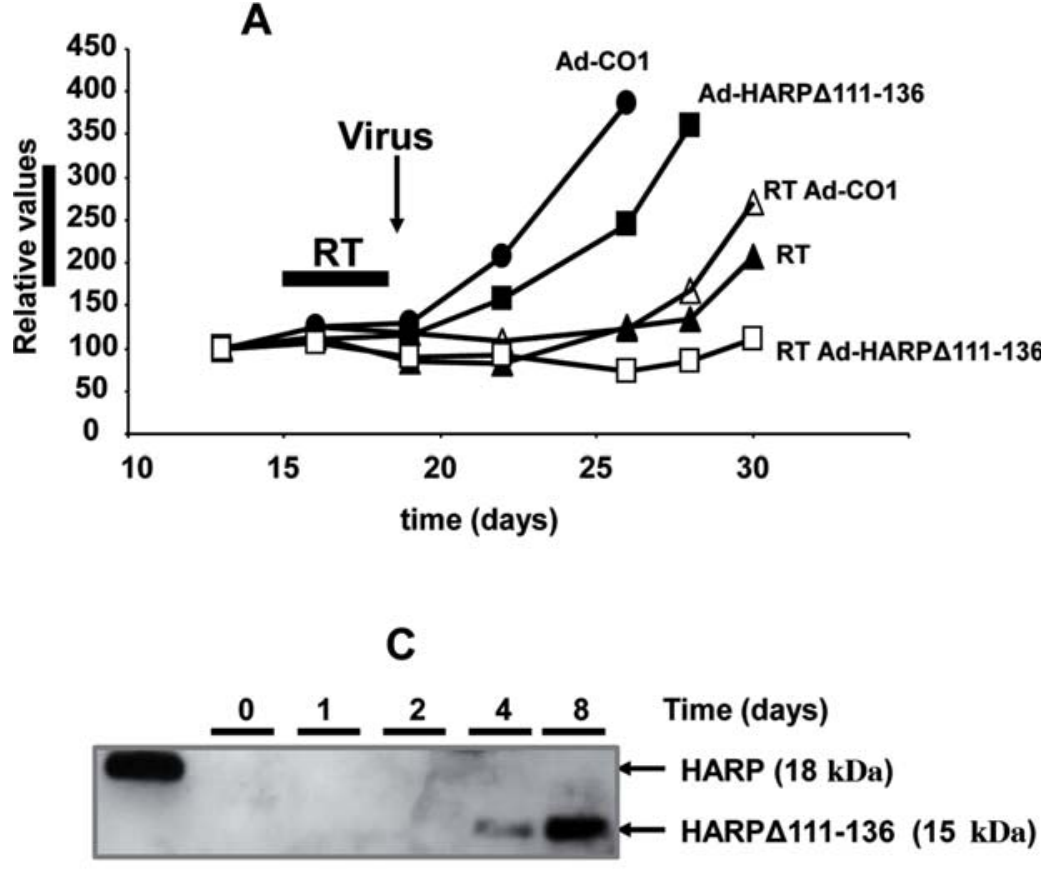

B

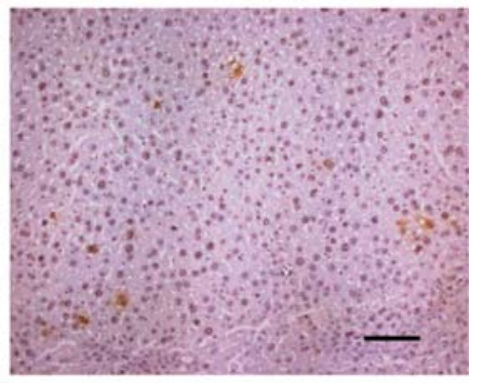

Ad-HARPA111-136

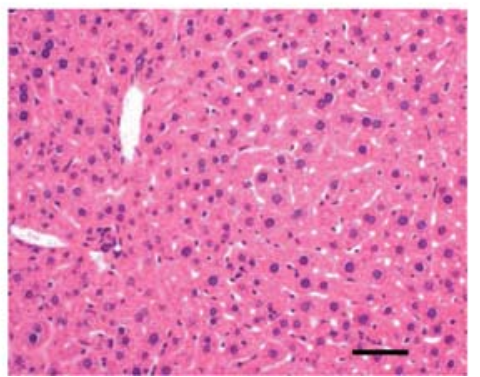

Figure 6. Systemic Ad-HARPA111-136 injection inhibited the growth of U87MG xenografts in vivo. Mice bearing U87MG subcutaneous xenografts were subjected or not to radiotherapy (2.5 Gy per day) on days 13,15 and 17 after the tumor graft. Viruses were injected intravenously (5x $10^{9}$ PFU) immediately after the last irradiation. (A) Effect of different treatments on U87MG tumor growth. Points represent the mean of volumes driven from at least 10 animals per group. (B) A representative illustration of liver sections derived from Ad-HARP $\Delta 111-136$ treated mice stained with hematoxylin-eosin-safran (lower panel) or immunohistochemically stained with an anti-HARP antibody (upper panel). Scale bar, $0.1 \mathrm{~mm}$. (C) Detection of peptide HARP $\Delta 111-136$ in mouse sera, after Ad-HARP $\Delta 111-136$ viral infection. The peptide HARPA111-136 (15 kDa) was concentrated on heparin-acrylic beads and analyzed by Western blotting using the anti-HARP antibody. Left line, recombinant $18 \mathrm{kDa}$ HARP was used as a positive control.

in vivo. To assess the in vivo effect of a bitherapy combining Ad-HARP $\Delta 111-136$ systemic injection with radiation therapy (RT), two million U87MG cells were subcutaneously injected into the back of nude mice. When mean tumor volumes reached $66 \pm 20 \mathrm{~mm}^{3}$, mice were randomized into five groups - Ad-CO1, Ad-HARPA111-136, RT, RT + Ad-CO1 and RT + Ad-HARP $\Delta 111-136$ (bi-therapy), and treatments were initiated. Three total body irradiations were performed on RT groups at a dose of 2.5 Gy at two days interval, starting on day 13 after tumor inoculation. Viruses were injected intravenously $\left(5 \times 10^{9} \mathrm{PFU}\right)$ immediately after the last irradiation. Our results demonstrated that on day 26, U87MG mean tumor volume (Fig. 6A) was significantly lower $(\mathrm{p}<0.01)$ in all groups that received a therapy compared to Ad-CO1 control group. There was no difference between groups receiving RT alone or RT + Ad-CO1 indicating that the viral infection itself had no influence on the effect of the radiation therapy. In accordance with our previous data using intra-tumoral AdHARP $\Delta 111-136$ injection (19), a single systemic injection of Ad-HARP $\Delta 111-136$ inhibited tumor growth by $40 \%(\mathrm{p}<0.01)$ when compared to control virus. A limited tumor growth was observed with the combination therapy, Ad-HARP $\Delta 111-136$ + RT. Furthermore, the bitherapy was significantly more efficient than each monotherapy (RT CO1 vs. RT+AdHARP $\Delta 111$-136: $\mathrm{p}=0.05$ on days 26 and $28, \mathrm{p}<0.01$ on day 30 ; Ad-HARP $\Delta 111-136$ vs. RT+Ad-HARP $\Delta 111-136: \mathrm{p}<0.001$ on days 26,28 and 30 ).
After systemic injection it is known that adenovirus infects the liver. To assess the in vivo peptide HARP $\Delta 111-136$ production after the viral infection, few mice were sacrificed during the first week after infection, their blood collected and their livers excised, fixed and processed for immunohistochemistry. The in vivo HARP $\Delta 111-136$ production was confirmed by anti-HARP immunostaining of liver sections as shown in Fig. 6B. Hematoxylin-eosin-safran staining of liver sections of all groups revealed a slight anisonucleosis related to a regeneration process without cytolysis.

Circulating HARP $\Delta 111-136$ in mice was concentrated from sera on heparin beads and in vivo HARP $\Delta 111-136$ production was assessed by Western blot analysis using antiHARP antibodies (Fig. 6C). The $15 \mathrm{kDa}$ protein (HARP $\Delta 111$ 136) appeared in mouse sera four days after Ad-HARP $\Delta 111$ 136 infection, and its level dramatically increased 8 days after the infection indicating a strong production of the HARP $\Delta 111$ 136 peptide in vivo.

The combination of RT and Ad-HARPAl11-136 treatments reduces tumor vessel density and triggers massive apoptosis of U87MG tumor cells in vivo. At the end of the experiment described before, U87MG tumors were excised and processed for immunohistological analysis. Tumor vascularization was assessed using anti-CD31 antibody (Fig. 7A). Large and thick tumor blood vessels, a feature of this highly angiogenic tumor, were only present in the Ad-CO1 control tumor 

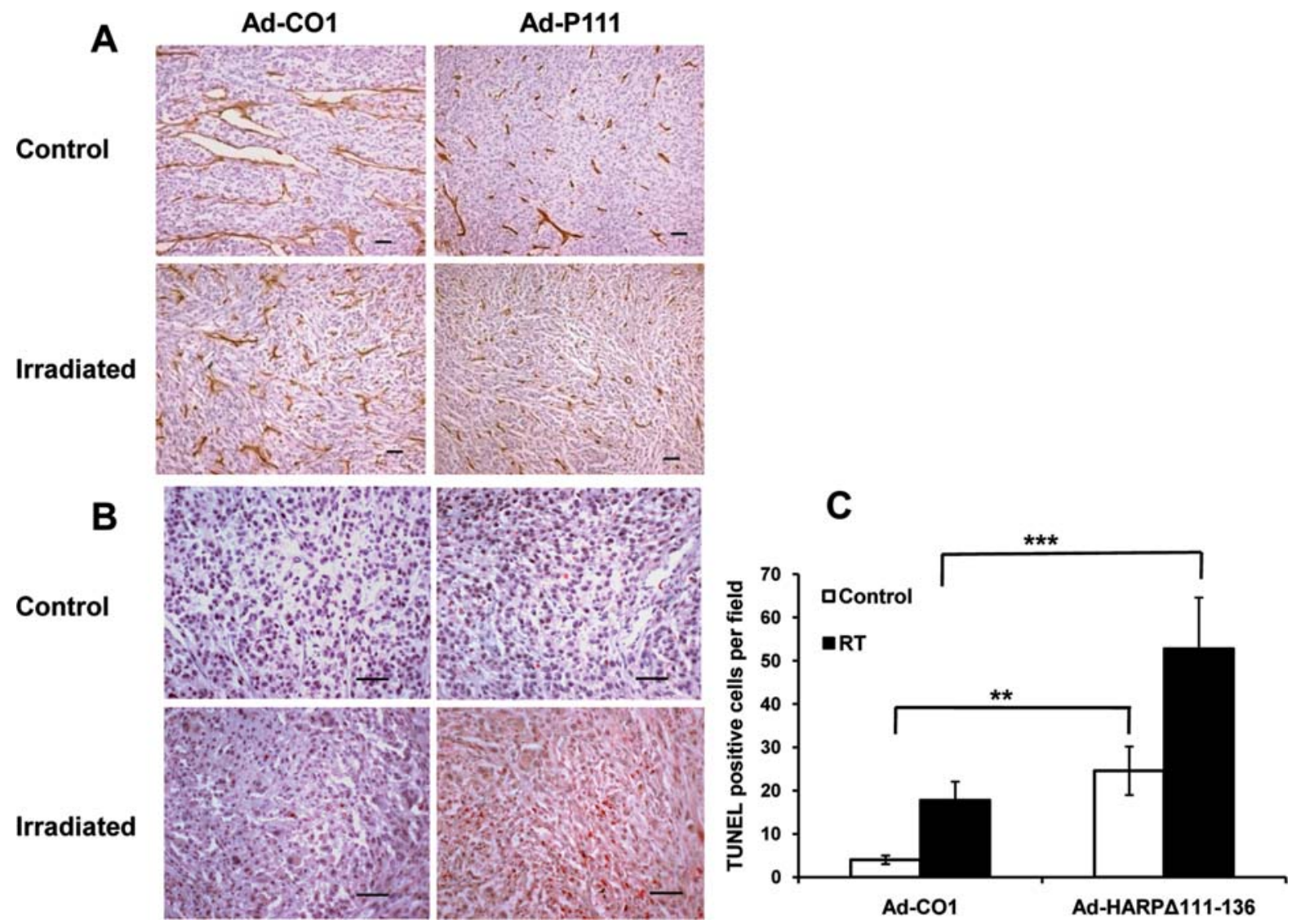

Figure 7. Anti-angiogenic and proapoptotic effects of Ad-HARPA111-136 on U87MG xenografts in vivo. The experiment protocol was the same as described in Fig. 6: eight days after virus infection, mice were sacrificed and their tumors were excised and processed for immunohistochemical analysis. (A) Tumor sections were stained with anti-CD31 antibody to visualize tumor vascularization. (B) Tumor sections were subjected to TUNEL assay to detect apoptosis. Apoptotic nuclei appear in red. (C) Five consecutive fields per tumors were digitized (200) and the number of positive cells per field was counted, the scoring was done in a double blinded fashion. Histograms represent the number of apoptotic nuclei per field for each experimental group. Values represent the mean of determinations performed on five experimental animals $\pm \mathrm{SD}$. Scale bar, $0.1 \mathrm{~mm} .{ }^{* *} \mathrm{p}<0.01,{ }^{* * *} \mathrm{p}<0.001$.

sections, whereas blood vessels in the Ad-HARP $\Delta 111-136$ group were much smaller in size and thinner. The normalized profile of blood vessels was constantly observed with the combination RT + Ad-HARP $\Delta 111-136$ therapy. The slides were scanned and the staining quantified using the automated PIXCYT software (26). The vascular density was significantly reduced $(30 \%, \mathrm{p}<0.001)$ in the group subjected to bitherapy when compared to RT + Ad-CO1, indicating that AdHARP $\Delta 111-136$ treatment counteracted the angiogenic effect of radiation therapy (32).

Apoptotic cells present in tumor sections were visualized in situ using TUNEL analysis. When compared with the AdCO1 control group (Fig. 7B), a single treatment by AdHARP $\Delta 111-136$ or RT significantly increased tumor U87MG cell apoptosis (Fig. 7C) by 6 -fold $(\mathrm{p}<0.01)$ and 4.5 -fold $(\mathrm{p}<0.05)$ respectively, while the combination of $\mathrm{RT}+\mathrm{Ad}-$ HARP $\Delta 111-136$ increased apoptosis by more than 13-fold $(\mathrm{p}<0.001 \%)$ compared to $\mathrm{RT}+\mathrm{Ad}-\mathrm{CO} 1$. The strong intensity of tumor apoptosis after the double treatment is illustrated by the background red color of the slides which was observed only in this group. These findings confirmed in vivo that the peptide HARPA111-136 sensitized U87MG cells to apoptosis.

\section{Discussion}

HARP has been described as a growth factor for several tumor types $(3,15,33)$ and in a previous study using U87MG glioblastoma cell line as an experimental model, we demonstrated that the HARP inhibitor HARP $\Delta 111-136$ exerts antiproliferative and potent anti-angiogenic activities. The aim of the present study was to investigate the mechanism of HARP $\Delta 111-136$ biological action; a recombinant adenovirus was used to produce high levels of the inhibitor HARP $\triangle 111-136$ in the glioblastoma U87MG cell line. Our results using RT-PCR and Western blotting demonstrated that the Ad-HARP $\Delta 111-136$ activated the ATF4/ATF3/CHOP pathway which is characteristic of the UPR response to ER stress. The ER stress involves three signaling pathways initiated by ATF6, PERK and IRE1 (21). Here we demonstrated the activation of two of these pathways in U87MG cells after infection with Ad-HARPA111-136. The increase in mRNA expression of ATF4 (CREB-2) and DDIT3 (CHOP) genes confirmed the activation of the PERK pathway. The activation of the IRE1 pathway was supported by the observed phosphorylation of JNK and P38 MAPK after Ad-HARP $\Delta 111-136$ infection. 
We focused our interest on the DDIT3 gene encoding the protein CHOP which was absent in steady state U87MG cells and was highly induced at mRNA and protein levels after Ad-HARP $\Delta 111-136$ treatment. The ER facilitates the intracellular trafficking of membrane and secreted proteins, however, when the capacity of the system is exceeded, the UPR is activated and CHOP is produced. Thus, in our experimental model CHOP could be simply induced by the protein overexpression after the viral infection. In order to verify this hypothesis U87MG cells were infected by recombinant adenovirus encoding either unsecreted GFP or a truncated form of angiostatin (K1-5). Like HARP $\Delta 111-136, \mathrm{~K} 1-5$, is a truncated and secreted protein. In both cases CHOP was not induced, thus, our results are consistent with a specific effect of HARP $\Delta 111-136$ on CHOP expression.

How HARP $\Delta 111-136$ activates ER stress is an important question that arises. We previously demonstrated that HARP $\Delta 111-136$ acts as a dominant negative effector of HARP by heterodimerizing with the wild-type HARP $(17,18)$. We hypothesized that expression of HARP $\Delta 111-136$ may indirectly lead to ER stress by preventing: i) HARP-induced oligomerization of the receptor $\mathrm{RPTP} \beta / \zeta$ and, ii) the downstream activation of protein kinase C (PKC) (29). This report demonstrated that Ad-HARP $\Delta 111-136$ reduced phosphorylation of PKC in U87MG cells. It is known from the literature that inhibition of PKC (30) leads to the rapid accumulation of B-catenin that activates early ER stress signaling via CHOP. Thus, we speculate that this mechanism is involved in HARP $\Delta 111-136$ effect. However, the interaction of the peptide HARP $\Delta 111-136$ with another intracellular pathway is not excluded and remains to be investigated.

Several studies suggest a potential role for CHOP in ER stress-induced neuronal cells death $(27,28,34)$. In the present study, the expression of CHOP after the Ad-HARP $\Delta 111-136$ infection was accompanied by a 10 -fold increase of apoptosis in U87MG cells. Apoptosis was confirmed by the activation of caspase 3 through the cleavage of its main substrate PARP. Furthermore, our in vitro study demonstrated that knockdown of CHOP using RNA interference considerably reduced the HARP $\Delta 111$-136-induced apoptosis, indicating a crucial role for CHOP in the downstream apoptotic effects of HARP $\Delta 111-136$. Our findings indicating that interruption of the HARP stimulation may lead cells to apoptosis are consistent with studies by Powers et al (33) who reported that targeting the HARP pathway in U87MG cells, using a ribozyme abolishing ALK expression, a HARP receptor, resulted in an increased apoptosis in U87MG xenografts. Other studies by Grzelinski et al (16) showed that knockdown of HARP using ribozymes induced apoptosis in U87MG cells.

CHOP is one of the critical effectors of the ER stress proapoptotic arm and activates the transcription of several genes that encode proapoptotic factors $(35,36)$. Our study demonstrated that infection of U87MG by Ad-HARP $\Delta 111-136$ resulted in an increased expression of the death receptor DR5 a downstream target of CHOP. It is known that upon activation by its ligand TRAIL, the death receptor DR5 mediates a potent proapoptotic effect $(37,38)$. The results of the present study demonstrated the extreme resistance of U87MG cells to TRAIL induced cell death, confirming observations by others (39). Our results demonstrated that Ad-HARP $\Delta 111-136$ enhanced
U87MG cell sensitivity to TRAIL-induced apoptosis, through up regulation of its receptor DR5 and subsequent cleavage of the downstream caspase 8 . These findings are in agreement with recent studies demonstrating that combining TRAIL with inducers of ER stress resulted in a strong sensitization to apoptosis of tumor cell lines (40) including several glioma cell lines $(41,42)$.

It is noteworthy that the adenoviral E1 and E3 genes encode proteins that specifically counteract apoptosis induced by death receptors (43). Despite deletion of these genes in our adenoviral construction, TRAIL-induced cell death was always higher in non-infected U87MG cells than in control Ad-CO1-infected cells (data not shown). Thus, in our study, the magnitude of the HARP $\Delta 111-136$ enhancing effect on TRAIL-induced apoptosis may have been underestimated.

Another way to induce tumor cell apoptosis is irradiation. Our data provide evidence, for the first time, that the peptide HARP $\Delta 111-136$ enhanced and even synergized in vitro with the effect of radiation therapy in U87MG cell line, through increased apoptosis of the tumor cells as analyzed by flow cytometry. This effect is CHOP-dependent as demonstrated by siRNA studies.

In our previous study (19) the anti-proliferative and antiangiogenic activities of HARP $\Delta 111-136$ were demonstrated in vivo on established subcutaneous glioblastoma U87MG tumors in which a single intra-tumoral injection of AdHARP $\Delta 111-136$ inhibited U87MG tumor growth by $45 \%$ and reduced tumor vessel density. In the present study, a single systemic Ad-HARPA111-136 injection in vivo also inhibited angiogenesis in subcutaneous glioblastoma U87MG tumors thus confirming the findings with intra-tumor injection. In the present study, the therapeutic molecule HARP $\Delta 111-136$ was produced in the liver causing minimal damage to hepatocytes as shown by the immunohistochemical analysis of liver sections, the molecule was drained into the blood as shown by Western blotting and finally acted distantly on the tumor. In vivo, TUNEL analysis of tumor sections provided evidence that the systemic Ad-HARP $\Delta$ 111-136 administration resulted in an increased tumor apoptosis and that, as illustrated in Fig. 7B, the combination of Ad-HARP $\Delta 111-136$ and radiation therapy (bitherapy) induced a massive tumor apoptosis.

In vivo tumor growth was strikingly inhibited during one month in the group subjected to bitherapy (Ad-HARP $\Delta 111$ $136+\mathrm{RT}$ ) owing to both antiangiogenic and proapoptotic effects as demonstrated by immunohistochemical studies. Statistical studies (Student's t-test) indicated that the bitherapy was significantly more efficient in reducing tumor growth than each monotherapy. Our results obtained with AdHARP $\Delta 111-136$ and irradiation are coherent with those of Yacoub et al (44) who reported in vitro radiosensitization of primary human glioblastoma cells with OSU-03012, a molecule known to induce the ER stress. Moreover, studies by Hideshima et al (45) showed that bortezomib, that induces ER stress, can restore the sensitivity of myeloma cells to chemotherapy induced apoptosis. As the peptide HARP $\Delta 111$ 136 sensitizes U87MG cells to the effects of both irradiation and TRAIL treatment in vitro, the combination of the three agents together may result in greater cell death and a tight control of tumors. 
In conclusion, our data emphasize the importance of CHOP signaling in apoptosis induced by the HARP inhibitor HARP $\Delta 111-136$. There is ample evidence showing that the peptide HARP $\Delta 111-136$ is a potent anti-angiogenic agent. Herein, we demonstrated a novel proapoptotic mechanism of action of this peptide on a glioblastoma cell line. Thus, due to its dual effect, inhibiting angiogenesis and, simultaneously, promoting apoptosis of the tumor cells, the peptide HARP $\Delta 111-136$ appears to be a promising multi-targeted pharmacological tool. Unlike glioblastoma, normal adult brain tissues do not express HARP and its two receptors (10), thus, tumor cells would be more susceptible to the HARP $\Delta 111-136$ cytotoxic effect than normal cells. A hallmark feature of U87MG cell line is an intense resistance to stimuli inducing apoptosis, therefore, HARP $\Delta 111-136$ could be exploited as a tool to sensitize cancer cells to standard therapy, with minimal potential toxicity.

\section{Acknowledgements}

Racha Karaky was funded by the Ministère Délégué à l'Enseignement Supérieur et à la Recherche. This study was supported in part by grants from ANR (ANR-06-RIB 016-02) and INCA (PL06-093), by the Centre National de la Recherche Scientifique and the Institut Gustave Roussy. We thank Olivia Bawa, Yann Lecluse, for their excellent technical assistance in immunohistochemistry and the flow cytometric studies.

\section{References}

1. Chauhan AK, Li YS and Deuel TF: Pleiotrophin transforms NIH 3T3 cells and induces tumors in nude mice. Proc Natl Acad Sci USA 90: 679-682, 1993

2. Li YS, Milner PG, Chauhan AK, Watson MA, Hoffman RM, Kodner CM, Milbrandt J and Deuel TF: Cloning and expression of a developmentally regulated protein that induces mitogenic and neurite outgrowth activity. Science 250: 1690-1694, 1990.

3. Fang W, Hartmann N, Chow DT, Riegel AT and Wellstein A: Pleiotrophin stimulates fibroblasts and endothelial and epithelial cells and is expressed in human cancer. J Biol Chem 267: 25889-25897, 1992

4. Laaroubi K, Delbe J, Vacherot F, Desgranges P, Tardieu M, Jaye M, Barritault D and Courty J: Mitogenic and in vitro angiogenic activity of human recombinant heparin affin regulatory peptide. Growth Factors 10: 89-98, 1994.

5. Maeda N, Nishiwaki T, Shintani T, Hamanaka H and Noda M: 6B4 proteoglycan/phosphacan, an extracellular variant of receptor-like protein-tyrosine phosphatase zeta/RPTPbeta, binds pleiotrophin/heparin-binding growth-associated molecule (HBGAM). J Biol Chem 271: 21446-21452, 1996.

6. Perez-Pinera P, Berenson JR and Deuel TF: Pleiotrophin, a multifunctional angiogenic factor: mechanisms and pathways in normal and pathological angiogenesis. Curr Opin Hematol 15: 210-214, 2008

7. Stoica GE, Kuo A, Aigner A, et al: Identification of anaplastic lymphoma kinase as a receptor for the growth factor pleiotrophin J Biol Chem 276: 16772-16779, 2001

8. Kadomatsu $\mathrm{K}$ and Muramatsu T: Midkine and pleiotrophin in neural development and cancer. Cancer Lett 204: 127-143, 2004.

9. Perez-Pinera P, Chang Y and Deuel TF: Pleiotrophin, a multifunctional tumor promoter through induction of tumor angiogenesis, remodeling of the tumor microenvironment, and activation of stromal fibroblasts. Cell Cycle 6: 2877-2883, 2007.

10. Mikelis C, Koutsioumpa M and Papadimitriou E: Pleiotrophin as a possible new target for angiogenesis-related diseases and cancer. Recent Pat Anticancer Drug Discov 2: 175-186, 2007.

11. Behin A, Hoang-Xuan K, Carpentier AF and Delattre JY: Primary brain tumours in adults. Lancet 361: 323-331, 2003.
12. Stupp R, Hegi ME, Gilbert MR and Chakravarti A: Chemoradiotherapy in malignant glioma: standard of care and future directions. J Clin Oncol 25: 4127-4136, 2007.

13. Furnari FB, Fenton T, Bachoo RM, et al: Malignant astrocytic glioma: genetics, biology, and paths to treatment. Genes Dev 21: 2683-2710, 2007

14. Ziegler DS, Kung AL and Kieran MW: Anti-apoptosis mechanisms in malignant gliomas. J Clin Oncol 26: 493-500, 2008.

15. Grzelinski M, Bader N, Czubayko F and Aigner A: Ribozymetargeting reveals the rate-limiting role of pleiotrophin in glioblastoma. Int J Cancer 117: 942-951, 2005.

16. Grzelinski M, Steinberg F, Martens T, Czubayko F, Lamszus K and Aigner A: Enhanced antitumorigenic effects in glioblastoma on double targeting of pleiotrophin and its receptor ALK. Neoplasia 11: 145-156, 2009.

17. Bernard-Pierrot I, Delbe J, Rouet V, Vigny M, Kerros ME, Caruelle D, Raulais D, Barritault D, Courty J and Milhiet PE: Dominant negative effectors of heparin affin regulatory peptide (HARP) angiogenic and transforming activities. J Biol Chem 277: 32071-32077, 2002.

18. Duces A, Karaky R, Martel-Renoir D, et al: 16-kDa fragment of pleiotrophin acts on endothelial and breast tumor cells and inhibits tumor development. Mol Cancer Ther 7: 2817-2827, 2008.

19. Santos CD, Karaky R, Renoir D, et al: Antitumorigenic effects of a mutant of the heparin affin regulatory peptide on the U87 MG glioblastoma cell line. Int J Cancer (In press).

20. Kim R, Emi M, Tanabe K and Murakami S: Role of the unfolded protein response in cell death. Apoptosis 11: 5-13, 2006.

21. Malhotra JD and Kaufman RJ: The endoplasmic reticulum and the unfolded protein response. Semin Cell Dev Biol 18: 716-731, 2007.

22. Heath-Engel HM, Chang NC and Shore GC: The endoplasmic reticulum in apoptosis and autophagy: role of the BCL-2 protein family. Oncogene 27: 6419-6433, 2008.

23. Puthalakath H, O'Reilly LA, Gunn P, et al: ER stress triggers apoptosis by activating BH3-only protein Bim. Cell 129: 1337-1349, 2007.

24. Galaup A, Magnon C, Rouffiac V, Opolon P, Opolon D, Lassau N, Tursz T, Perricaudet M and Griscelli F: Full kringles of plasminogen (aa 1-566) mediate complete regression of human MDA-MB-231 breast tumor xenografted in nude mice. Gene Ther 12: 831-842, 2005.

25. Magnon C, Opolon P, Connault E, Mir LM, Perricaudet M and Martel-Renoir D: Canstatin gene electrotransfer combined with radiotherapy: preclinical trials for cancer treatment. Gene Ther 15: 1436-1445, 2008.

26. Elie N, Plancoulaine B, Signolle JP and Herlin P: A simple way of quantifying immunostained cell nuclei on the whole histologic section. Cytometry A 56: 37-45, 2003.

27. Zinszner H, Kuroda M, Wang X, Batchvarova N, Lightfoot RT, Remotti H, Stevens JL and Ron D: CHOP is implicated in programmed cell death in response to impaired function of the endoplasmic reticulum. Genes Dev 12: 982-995, 1998.

28. Tajiri S, Yano S, Morioka M, Kuratsu J, Mori M and Gotoh T: $\mathrm{CHOP}$ is involved in neuronal apoptosis induced by neurotrophic factor deprivation. FEBS Lett 580: 3462-3468, 2006.

29. Pariser H, Herradon G, Ezquerra L, Perez-Pinera P and Deuel TF: Pleiotrophin regulates serine phosphorylation and the cellular distribution of beta-adducin through activation of protein kinase C. Proc Natl Acad Sci USA 102: 12407-12412, 2005.

30. Raab MS, Breitkreutz I, Tonon G, et al: Targeting PKC: a novel role for beta-catenin in ER stress and apoptotic signaling. Blood 113: 1513-1521, 2009.

31. Zou CG, Cao XZ, Zhao YS, Gao SY, Li SD, Liu XY, Zhang Y and Zhang KQ: The molecular mechanism of endoplasmic reticulum stress-induced apoptosis in PC-12 neuronal cells: the protective effect of insulin-like growth factor I. Endocrinology 150: 277-285, 2009.

32. Gorski DH, Beckett MA, Jaskowiak NT, et al: Blockage of the vascular endothelial growth factor stress response increases the antitumor effects of ionizing radiation. Cancer Res 59: 3374-3378, 1999.

33. Powers C, Aigner A, Stoica GE, McDonnell K and Wellstein A: Pleiotrophin signaling through anaplastic lymphoma kinase is rate-limiting for glioblastoma growth. J Biol Chem 277: 14153-14158, 2002 .

34. Yamaguchi H and Wang HG: CHOP is involved in endoplasmic reticulum stress-induced apoptosis by enhancing DR5 expression in human carcinoma cells. J Biol Chem 279: 45495-45502, 2004. 
35. Zhang $\mathrm{K}$ and Kaufman RJ: From endoplasmic-reticulum stress to the inflammatory response. Nature 454: 455-462, 2008.

36. Ohoka N, Yoshii S, Hattori T, Onozaki K and Hayashi H: TRB3, a novel ER stress-inducible gene, is induced via ATF4-CHOP pathway and is involved in cell death. EMBO J 24: 1243-1255, 2005 .

37. Johnstone RW, Frew AJ and Smyth MJ: The TRAIL apoptotic pathway in cancer onset, progression and therapy. Nat Rev Cancer 8: 782-798, 2008

38. Oldenhuis CN, Stegehuis JH, Walenkamp AM, de Jong S and de Vries EG: Targeting TRAIL death receptors. Curr Opin Pharmacol 8: 433-439, 2008.

39. Hao C, Beguinot F, Condorelli G, Trencia A, Van Meir EG Yong VW, Parney IF, Roa WH and Petruk KC: Induction and intracellular regulation of tumor necrosis factor-related apoptosisinducing ligand (TRAIL) mediated apotosis in human malignant glioma cells. Cancer Res 61: 1162-1170, 2001.

40. Taniguchi H, Yoshida T, Horinaka M, Yasuda T, Goda AE, Konishi M, Wakada M, Kataoka K, Yoshikawa T and Sakai T: Baicalein overcomes tumor necrosis factor-related apoptosisinducing ligand resistance via two different cell-specific pathways in cancer cells but not in normal cells. Cancer Res 68: 8918-8927, 2008.
41. Koschny R, Holland H, Sykora J, et al: Bortezomib sensitizes primary human astrocytoma cells of WHO grades I to IV for tumor necrosis factor-related apoptosis-inducing ligand-induced apoptosis. Clin Cancer Res 13: 3403-3412, 2007.

42. Hetschko H, Voss V, Seifert V, Prehn JH and Kogel D: Upregulation of DR5 by proteasome inhibitors potently sensitizes glioma cells to TRAIL-induced apoptosis. FEBS J 275: 1925-1936, 2008.

43. McNees AL and Gooding LR: Adenoviral inhibitors of apoptotic cell death. Virus Res 88: 87-101, 2002.

44. Yacoub A, Park MA, Hanna D, et al: OSU-03012 promotes caspase-independent but PERK-, cathepsin B-, BID-, and AIFdependent killing of transformed cells. Mol Pharmacol 70: 589-603, 2006.

45. Hideshima T, Richardson P, Chauhan D, Palombella VJ Elliott PJ, Adams J and Anderson KC: The proteasome inhibitor PS-341 inhibits growth, induces apoptosis, and overcomes drug resistance in human multiple myeloma cells. Cancer Res 61: 3071-3076, 2001. 